\section{UNIQUE CASE OF FOREIGN BODY IN THE BLADDER.}

J. F. BALDWIN, M.D.

Surgeon to Grant Hospital.

COLUMBUS, OHIO.

While the literature of foreign bodies in surgery is voluminous and interesting, the case detailed below is, so far as my investigation of the literature goes, entirely unique, especially as regards the snare devised by the patient for the removal of the foreign body.

History.-An old soldier, aged 70, for several years had been troubled with prostatic retention of urine. For about a year he used a catheter, which while it answered the purpose fairly well, was not entirely satisfactory. He therefore took a piece of steel rod, about two inches long, rounded it at one end and flattened it at the other, and through a perforation in the flattened end passed a fine wire to be used in withdrawing the device, which, as thus made, very much resembled a sinker, such as boys sometimes use in fishing. He had a capacious urethra, and by lying down and allowing this sinker to drop into the deep urethra and into the neck of the bladder, the obstruction was so displaced that he was able to urinate without difficulty. He found the device exceedingly satisfactory and had used it for about-a year when, unfortunately, the wire rusted where it passed through the hole in the sinker, and the latter was left in the bladder. The wire had been purely for the purpose of withdrawal, since he relied on the weight of the sinker for its passage into the bladder. To remove the metal, and without consulting a physician, the patient bent a wire into an elongated loop, as shown in the cut. He had a piece of steel from which he had made his original sinker, and in bending his loop he so made it that when the

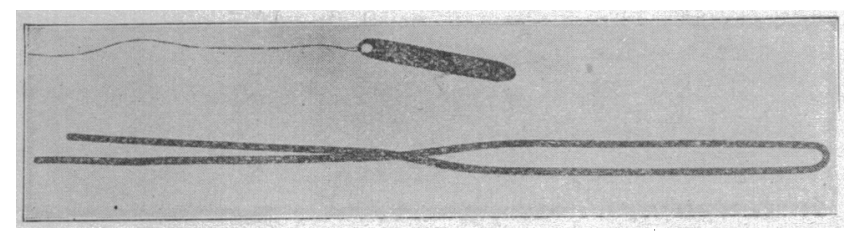

ends of the wire were approximated the sinker might engage in the loop and then be caught by separating them. With great fortitude he introduced this loop into the bladder, and spent considerable time in a fruitless attempt to snare the sinker. He finally consulted his physician, Dr. Kious, through whom the case was referred to me for operative intervention.

operation.-An ordinary suprapubic cystotomy was performed Dec. 9, 1905, through which the piece of steel was easily removed. The obstruction was found to consist in a transverse bar in the floor of the internal meatus. This bar was cut across with scissors, and the surface touched with the electric cautery-a sort of transvesical Bottini. Recovery was prompt and entirely satisfactory.

The accompanying illustration shows the sinker and snare, one-third actual size.

\section{Special Article}

\section{SOME FACTS ABOUT DIGESTIVE FERMENTS. TORALD SOLLMANN, M.D. CLEVELAND, OHIO. \\ (Continued from page 416.)}

OTHER INCOMPATIBILITIES IN LIQUID DIGESTANTS.

The compound digestive liquids do not constitute the only incompatibility in the manufacture or prescription of digestive ferments, although they are probably the most glaring example. We must again recall that ferments are peculiarly unstable bodies; indeed, there are few substances so sensitive to reagents, so liable to injury, as the ferments, especially when they are dissolved.
Acids and alkalies, salts, metals, alkaloids, tannins, antiseptics, chloroform, ether and alcohol-all these interfere more or less with the action of ferments and injure their activity. Alcohol is especially important, since it is a conspicuous constituent of the digestive "wines" and elixirs, particularly those of pepsin.

\section{THE EFFECT OF ALCOHOL ON FERMENTS.}

A short contact with alcohol does not appear to be injurious to most ferments, since one of the ordinary methods of purifying the crude ferments consists in their precipitation by alcohol. Prolonged contact with alcohol, however, even when quite dilute, causes a gradual destruction. This deterioration is a rather slow process, especially with pepsin, so that there may be no great objection to prescribing a freshly made wine or elixir of pepsin when it is to be used in a few days. The proprietary preparations, however, are generally made a long time before they are administered, and their activity must be very unreliable. As a matter of fact, the preparations of this class examined by the Council proved quite worthless from the digestive standpoint, and the popularity of certain proprietary compounds must be attributed solely to their seductive taste and appearance.

\section{GLYCERIN AS A PRESERVATIVE.}

Aqueous solutions of the ferments also deteriorate very rapidly. The only liquid medium in which ferments can be preserved for any length of time is glycerin. In this connection it may be pointed out that the advantage of glycerins of pepsin made by extracting the mucous membrane directly with glycerin, over those made by dissolving dried, soluble pepsin in glycerin, is not very apparent. A carefully made glycerin extract would certainly be better than a cheap glycerin solution, for the glycerin extraction precludes putrefactive changes; but the careful manufacturer of pepsin will have no difficulty in avoiding putrefaction. It may be true that the glycerin extract contains more milkcurdling ferment and is superior for the manufacture of junket. For internal administration, however, this has no significance. Pepsin is used to digest proteids, and this power is possessed by the dry pepsin and by its solution in glycerin. If there are any therapeutic indications for other ingredients of the gastric juice (aside from the acid) they have not yet been formulated. As to the rennin, the most authoritative book dealing with ferments (Oppenheimer) says as follows:

It does not appear to have any essential significance for the digestion of proteids. It is strikingly absent in the newborn, who consume much milk. Zuntz and Sternberg have even found that milk proteid coagulated by rennin is less easily digested than the original milk proteid, and attribute to this in part the relatively smaller availability of milk for adults, who produce more rennin.

\section{DRY MIXTURES OF FERMENTS.}

Since the digestive ferments act only in solution, it might be thought that the object of combining pepsin, trypsin and diastase could be attained by administering the mixtures as dry powders or tablets. These will, indeed, keep indefinitely if they are protected from moisture; but a moment's thought will show that their destruction will begin with their action. The conditions for this will arise the moment that they enter into solution in the mouth or stomach. This brings us to the question as to how far ferments, artificially introduced by mouth, may be effective in the alimentary canal. 